\section{Soft nanocomposite electroadhesives for digital micro- and nanotransfer printing}

\author{
Sanha Kim ${ }^{1,2}$, Yijie Jiang ${ }^{3}$, Kiera L. Thompson Towell ${ }^{3}$, Michael S. H. Boutilier ${ }^{1}$, \\ Nigamaa Nayakanti ${ }^{1}$, Changhong Cao ${ }^{1}$, Chunxu Chen ${ }^{3}$, Christine Jacob ${ }^{1}$, Hangbo Zhao ${ }^{1}$, \\ Kevin T. Turner ${ }^{3}$, A. John Hart ${ }^{1 *}$
}

\begin{abstract}
Automated handling of microscale objects is essential for manufacturing of next-generation electronic systems. Yet, mechanical pick-and-place technologies cannot manipulate smaller objects whose surface forces dominate over gravity, and emerging microtransfer printing methods require multidirectional motion, heating, and/or chemical bonding to switch adhesion. We introduce soft nanocomposite electroadhesives (SNEs), comprising sparse forests of dielectric-coated carbon nanotubes (CNTs), which have electrostatically switchable dry adhesion. SNEs exhibit 40 -fold lower nominal dry adhesion than typical solids, yet their adhesion is increased $>100$-fold by applying $30 \mathrm{~V}$ to the CNTs. We characterize the scaling of adhesion with surface morphology, dielectric thickness, and applied voltage and demonstrate digital transfer printing of films of Ag nanowires, polymer and metal microparticles, and unpackaged light-emitting diodes.
\end{abstract}

\section{INTRODUCTION}

Continued advances in electronic devices demand the heterogeneous integration and assembly of components with ever-smaller dimensions. For instance, mechanical sensors including multiaxis inertial sensors and accelerometers are smaller than a millimeter (1), integrated circuit (IC) chips for radio-frequency communication are $0.1 \mathrm{~mm}$ or smaller (2), and micro-light-emitting diodes (LEDs) for next-generation displays are 1 to $10 \mu \mathrm{m}$ in lateral size (3). Furthermore, functional systems for applications in energy conversion, optoelectronics, and biotechnology (4) can be realized by manipulation of complex three-dimensional (3D) micro- and nanomaterials.

At present, automated pick-and-place assembly is the benchmark technique for manufacturing of circuit boards and integration of miniature ICs into systems-on-chip (5-7). However, despite advances in miniature robotic grippers and vacuum nozzles, manipulation is increasingly challenging as object size decreases, because gravity forces diminish more rapidly than van der Waals forces or electrostatic attraction (8). Intricate micromechanical grippers suffer from stiction and therefore cannot reliably place microscale objects without the aid of secondary adhesive surfaces $(9,10)$. Therefore, manipulation devices with surfaces that can repeatedly switch between strong adhesion for object pickup and weak adhesion for object placement are necessary for printing at smaller scales. Heterogeneous integration without direct physical manipulation may be possible by self-assembly methods $(11,12)$, yet precise dimensional control and long-range accuracy of self-assembly are challenging.

Engineering a tunable adhesive surface requires control of both the nanoscale contact mechanics and a reversible mechanism by which attractive forces can be varied between the surface and the target object, ideally without requiring application of shear (13-15), heating (16), or chemical reactions $(17,18)$. To realize such functionality, we introduce a soft nanocomposite (Fig. 1A) comprising self-assembled nanofibers that, by electrically switchable adhesion, can achieve digi-

\footnotetext{
'Department of Mechanical Engineering, Massachusetts Institute of Technology, Cambridge, MA, USA. ${ }^{2}$ Department of Mechanical Engineering, Korea Advanced Institute of Science and Technology, Daejeon, South Korea. ${ }^{3}$ Department of Mechanical Engineering and Applied Mechanics, University of Pennsylvania, Philadelphia, PA, USA.

*Corresponding author. Email: ajhart@mit.edu
}

tally controllable transfer printing of micro- and nanoscale objects. The soft nanocomposite electroadhesive (SNE) has a very low intrinsic ("off") dry adhesion through roughness and reduced contact area, while voltage-tunable electrostatic attraction gives strong "on" adhesion force that can overcome the intrinsic adhesion of micro- and nanostructures against donor substrates. The SNE is distinct from well-known electrostatic grippers and electroadhesive films (19-21), as the surface is composed of sparse nanofibers that are nonadhesive yet can be highly compressed. The SNE can therefore conformally contact miniature objects in the absence of an electrostatic force, without adhering. The nanocomposite fibers are conductive but covered with ultrathin dielectric, thus allowing substantial electrostatic pressures to be achieved at low operating voltages $(0$ to $100 \mathrm{~V})$.

We designed and fabricated SNEs (Fig. 1B) comprising "forests" of vertically aligned multiwalled carbon nanotubes (CNTs; $~ 10 \mathrm{~nm}$ in diameter) $(22,23)$, grown on a conductive electrode [titanium nitride (TiN), $50 \mathrm{~nm}$ in thickness] (24), and then conformally coated with a thin layer of dielectric $\left(\mathrm{Al}_{2} \mathrm{O}_{3}, \sim 1\right.$ to $10 \mathrm{~nm}$ in thickness) by atomic layer deposition (ALD) (25). Further details are given in Materials and Methods and fig. S1. An exemplary pick-and-place sequence using a single SNE pixel is shown in Fig. 1C (movie S1), whereby a $170 \mu \mathrm{m}$ by $170 \mu \mathrm{m}$ by $50 \mu \mathrm{m}$ unpackaged LED chiplet (CREE; detailed geometry in fig. S2) is lifted by the SNE with $30 \mathrm{~V}$ applied and placed with $0 \mathrm{~V}$ applied.

\section{RESULTS AND DISCUSSION}

To study the electrical tunability of adhesion, we measured the adhesive forces of the $\mathrm{Al}_{2} \mathrm{O}_{3}$-CNT SNE at different applied voltages using atomic force microscopy (AFM) with a Pt-coated spherical tip $(2 \mu \mathrm{m}$ in radius), which emulates a representative microscale object (Fig. 1D; see fig. S3 for further details). The adhesion, measured by the pulloff force of the cantilever against the SNE, scales quadratically with applied voltage. The off-state adhesion of the $\mathrm{SNE}$ is measured to be $\sim 40$-fold lower than a reference flat surface $\left(\mathrm{ALD} \mathrm{Al}_{2} \mathrm{O}_{3}\right.$ on coated flat TiN on a Si wafer), yet the SNW adhesion increases by $>100$-fold when $30 \mathrm{~V}$ is applied. In micromanipulation, this low off-state adhesion is important to allow objects to be deterministically released from the gripping surface, while the high on-state adhesion enables objects to 
A

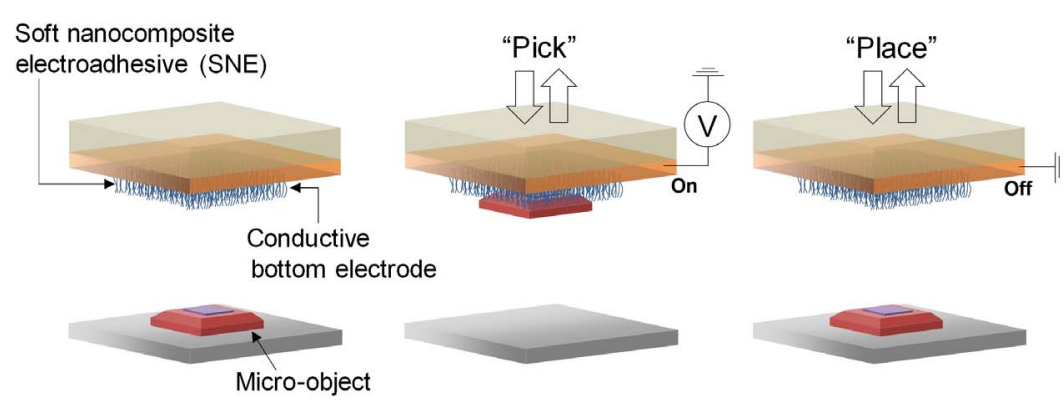

B Uniform, ultrathin $\mathrm{Al}_{2} \mathrm{O}_{3}$ coating

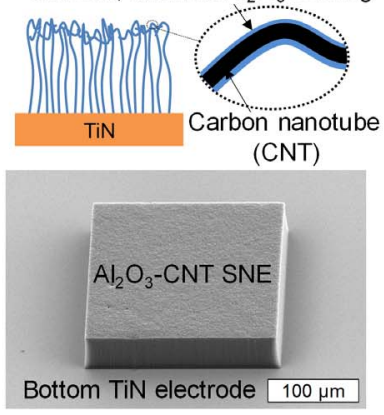

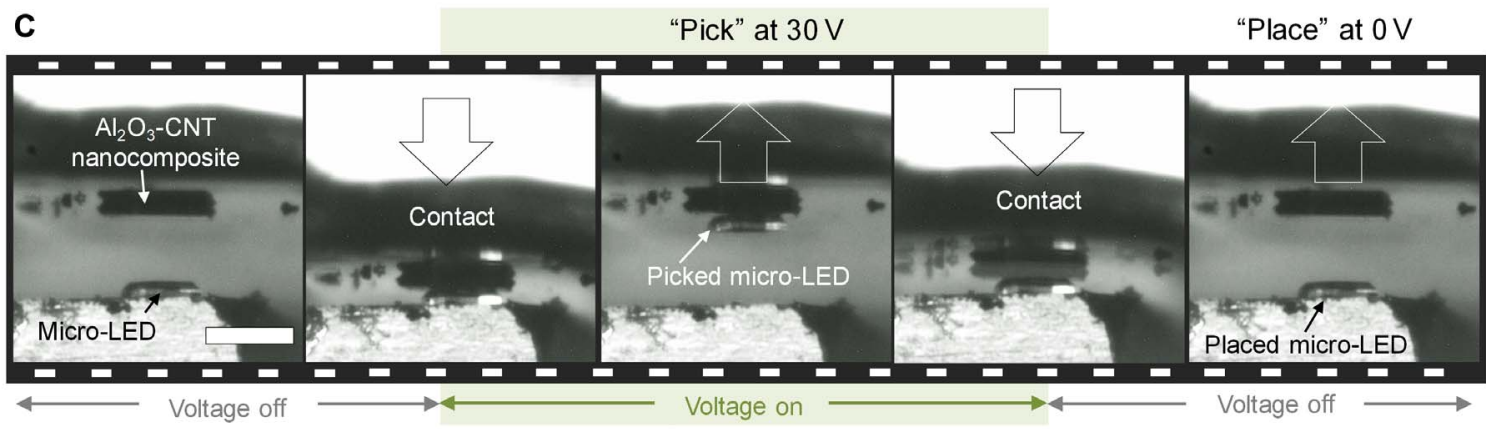

D
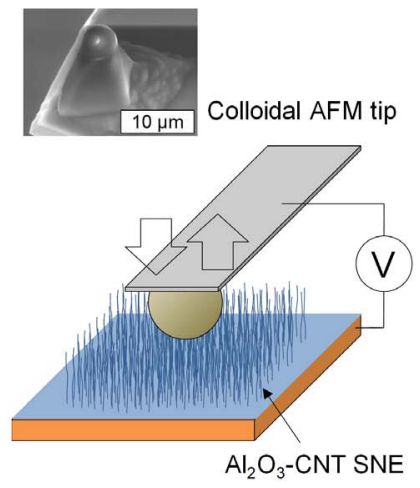

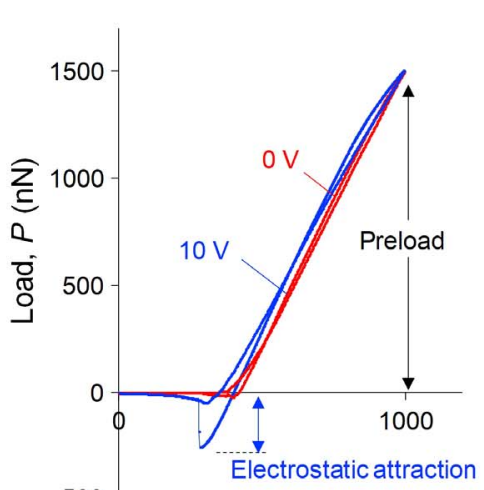

Displacement, $\delta(\mathrm{nm})$

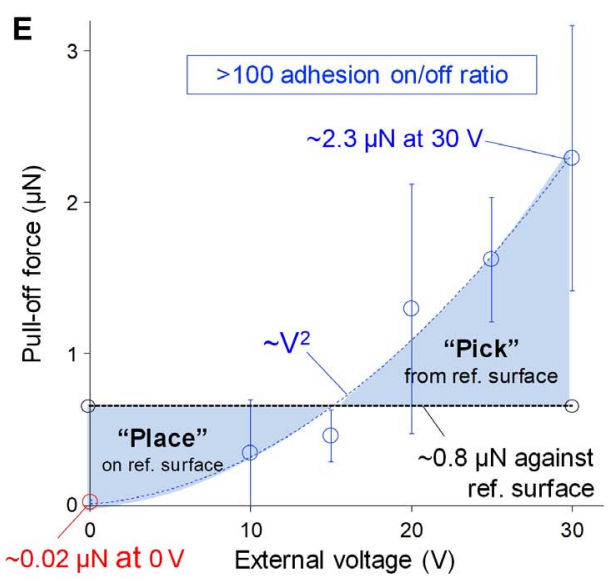

Fig. 1. Picking and placing micro objects using a ceramic-carbon SNE. (A) Schematic of a pick-and-place procedure wherein a micrometer-sized object in contact with an SNE surface can be picked up, while an external voltage is applied and placed back when the voltage is removed. (B) Schematics and scanning electron microscopy (SEM) images of a 200- $\mu \mathrm{m}$ SNE composed of $\mathrm{Al}_{2} \mathrm{O}_{3}$-coated carbon nanotubes (CNTs). (C) Snapshots of picking and placing an unpackaged LED chiplet $(170 \mu \mathrm{m}$ by $170 \mu \mathrm{m}$ by $50 \mu \mathrm{m})$ using the $\mathrm{Al}_{2} \mathrm{O}_{3}$-CNT nanocomposite electroadhesive ( $200 \mu \mathrm{m}$ by $200 \mu \mathrm{m}$ by $50 \mu \mathrm{m}$ ). (D) Schematic of pull-off force measurement system using a colloidal AFM tip and load-displacement curves for a $\mathrm{Al}_{2} \mathrm{O}_{3}-\mathrm{CNT}$ SNE measured using a $\sim 4-\mu \mathrm{m}$-diameter Pt-coated spherical tip with and without a bias voltage of $10 \mathrm{~V}$. The inset SEM image shows the Pt-coated spherical tip. (E) Measured pull-off forces using the Pt-coated spherical tip against a reference surface (flat $\mathrm{Al}_{2} \mathrm{O}_{3}$-coated TiN; $\sim 0.8 \mu \mathrm{N}$; black dashed line) and against the $\mathrm{Al}_{2} \mathrm{O}_{3}$ - CNT SNE at different applied voltages (e.g., $\sim 0.02 \mu \mathrm{N}$ at $0 \mathrm{~V}$ and $\sim 2.3 \mu \mathrm{N}$ at $30 \mathrm{~V}$; blue dashed line), measured by a conductive AFM. Error bars represent the SD in the set of force measurements with each applied voltage.

be picked from donor substrates that have high intrinsic adhesion to the object (Fig. 1E). Here, we found greater measurement errors using the Pt-coated tips compared to those using uncoated tips. Using a spherical AFM tip comprising a metal sphere directly adhered to a conductive cantilever may improve the measurement accuracy.

The low off-state adhesion of the SNE is due to its low-density fibrous texture and nanoscale roughness, both of which contribute to a low effective contact area against an opposite surface. The selforganization of the CNTs during the CVD process imparts the forest with a top surface consisting of randomly oriented CNTs, supported by well-aligned CNTs underneath (Fig. 2A) (26). As the fibrous SNE makes contact with an object, the CNT forest behaves as a soft foam, where the surface deformation is accompanied by the spring-like behavior of the supporting aligned CNTs (Fig. 2B). The roughness of the $\mathrm{Al}_{2} \mathrm{O}_{3}$-CNT SNE surface measured by AFM is approximately $35 \mathrm{~nm}$ (Fig. 2C), and the reduced modulus measured by nanoindentation is $86 \mathrm{MPa}$, which is $\sim 1000$-fold less than the flat surface reference (Fig. 2D). Hence, while the surface can deform easily and conformally contact micro- and nanoscale objects with $3 \mathrm{D}$ shapes, only a few protruding nanocomposite fibers of the SNE surface will effectively be in 
A

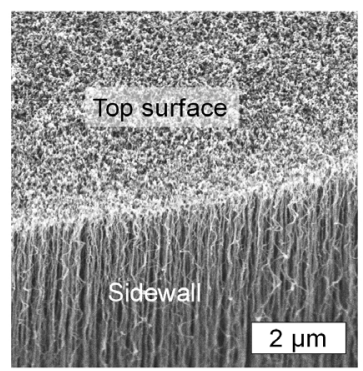

C
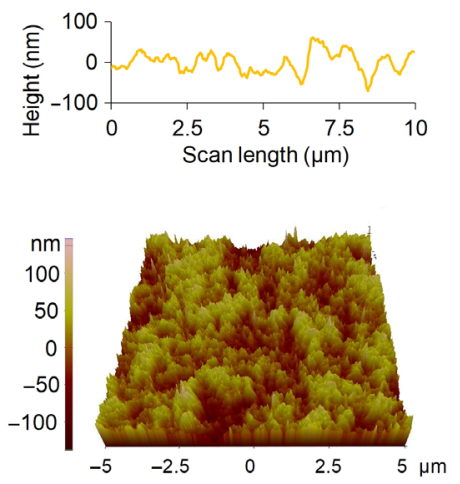

B

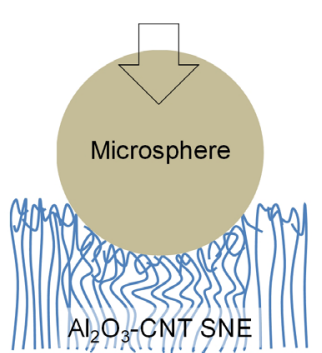

Spherical tip

D

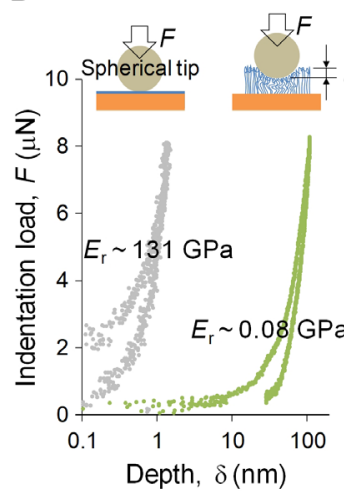

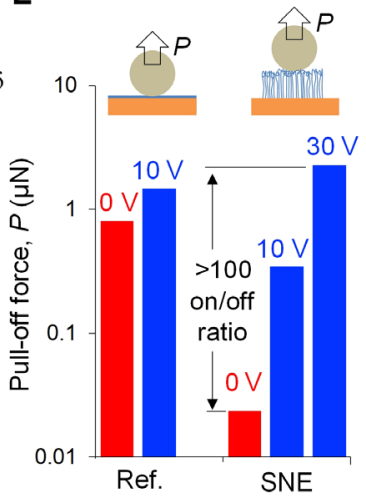

$\mathbf{F}$

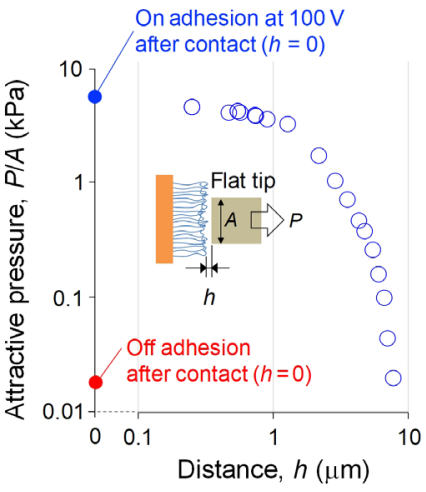

Fig. 2. Fibrous structure, roughness, and softness of SNE surface enabling switchable adhesion. (A) An SEM image of SNE showing the top entangled surface and vertically aligned fibers underneath. (B) Schematics showing structural behavior of the top surface when a microsphere is compressed and retracted (left) and showing the van der Waals force that scales with few contacting nanofibers and electrostatic attraction that scales with all the nanofibers on the SNE surface (right). (C) AFM images of the top SNE surface composed of entangled $\mathrm{Al}_{2} \mathrm{O}_{3}$-coated CNT fibers. The root mean square roughness within the $100-\mu \mathrm{m}^{2}$ area is $\sim 35 \mathrm{~nm}$. (D) Load displacement of $\mathrm{Al}_{2} \mathrm{O}_{3}$-coated surfaces with and without $\mathrm{SNE}$, measured by nanoindentation using a $\sim 10$ - $\mu$ m-diameter sapphire tip. (E) Pull-off forces of $\mathrm{Al}_{2} \mathrm{O}_{3}$-coated surfaces with and without SNE measured by AFM using the $\sim 4-\mu \mathrm{m}$-diameter Pt-coated spherical tip. (F) Attractive pressure at distances from the $\mathrm{Al}_{2} \mathrm{O}_{3}-\mathrm{CNT}$ SNE surface measured using the $1.2 \mathrm{~mm}$ by $1.2 \mathrm{~mm}$ Au-coated flat tip.

contact at the interface. Only these contacting fibers contribute to the surface adhesion during retraction, as the surrounding noncontacting fibers do not contribute to the van der Waals adhesion.

The on-state adhesion is determined by electrostatic charge accumulated on the nanofibers both in contact with and in proximity of the object surface, because the electrostatic forces are effective at distances greater than van der Waals forces. As a result, the electrostatic attraction of the SNE to an object can be significantly greater than the van der Waals attraction, especially for rough surfaces. This contrasts the behavior of smooth and flat surfaces, as the van der Waals forces are comparable with the electrostatic forces unless very high voltages $(>1 \mathrm{kV})$ are applied. Accordingly, for the rigid flat surface, the ratio of the on-state adhesive force (with voltage applied) to the off-state $(0 \mathrm{~V})$ force is only $\sim: 1$ at $30 \mathrm{~V}$, yet the on/off ratio of the $\mathrm{Al}_{2} \mathrm{O}_{3}$-CNT SNE is $\sim 100: 1$ at the same voltage (Fig. 2E and fig. S4). For the SNE, this 100:1 ratio is maintained with contact and at up to $\sim 1-\mu \mathrm{m}$ separation distance, as shown in the measurements of Fig. $2 \mathrm{~F}$, which were performed using a $1.2 \mathrm{~mm}$ by $1.2 \mathrm{~mm}$ flat square tip with $100 \mathrm{~V}$ applied.

Such a long-range attraction is advantageous for picking objects with porous and/or rough surfaces. For example, we demonstrate picking and placing a piece of paper towel that cannot be manipulated using an elastomer stamp such as polydimethylsiloxane (movie S2), because the microscopically porous and rough surface (fig. S5) cannot achieve enough viscoelastic adhesion from the small physical contact area. In contrast, SNE can attract both smooth and rough surfaces because of its compliance and the long-range electrostatic attraction.

Apart from controlling the electrostatic charge at the interface, the on-state adhesive strength can be tailored by the material properties and geometry of the hierarchical SNE surface. For example, we find that the dielectric coating thickness, CNT forest density (i.e., fibers per unit area), and the resulting mechanical compliance are the key design parameters for effective SNEs with maximum on/off ratio (Fig. 3A). Surfaces with thinner dielectric coatings exhibit high on/off ratios by reducing the distance between the oppositely charged CNTs and the object surface. A subnanometer $\mathrm{Al}_{2} \mathrm{O}_{3}$ coating $(\sim 0.2 \mathrm{~nm})$ can achieve higher electrostatic attraction forces at a given voltage, although the nonuniformity of such a thin coating (fig. S6), even with ozone-assisted ALD (27), often resulted in breakdown at low voltages. Thus, we found that the minimum thickness conformal dielectric coating was at least $\sim 1 \mathrm{~nm}$. In addition, the density and morphology of the CNT forest surface can be tailored by the CVD growth recipe. For example, a CNT forest with high fiber density exhibits a greater adhesion on/off ratio than a CNT forest with a low fiber density (fig. S7). The electroadhesive properties are independent of forest height beyond $\sim 1 \mu \mathrm{m}$ (fig. S8), and therefore, the SNE can be designed with fine features by lithographic patterning of the CNTs, or even with spatially varying heights and complex free-form microstructured shapes $(28,29)$. 
A

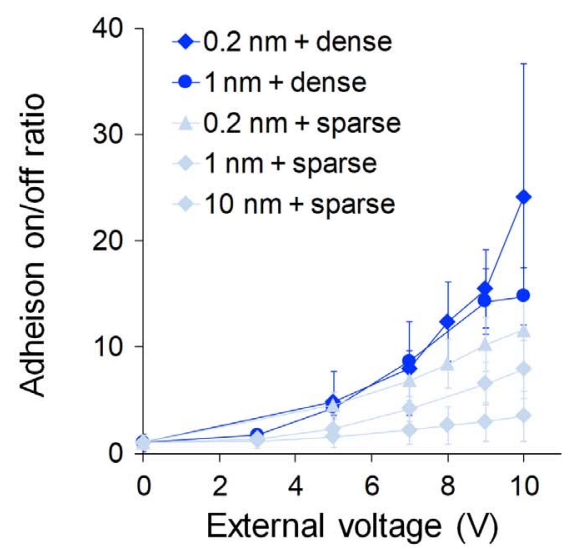

C

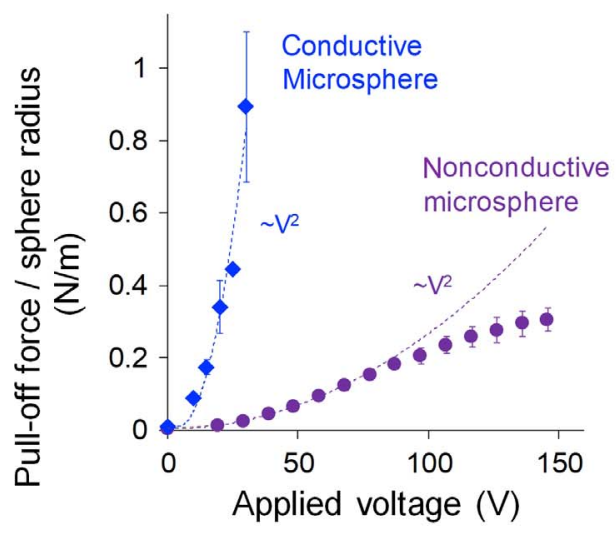

B
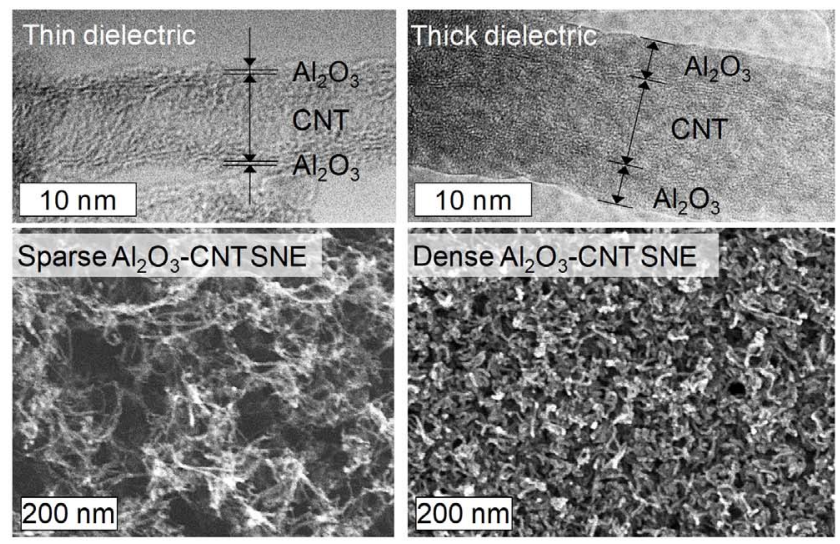

D

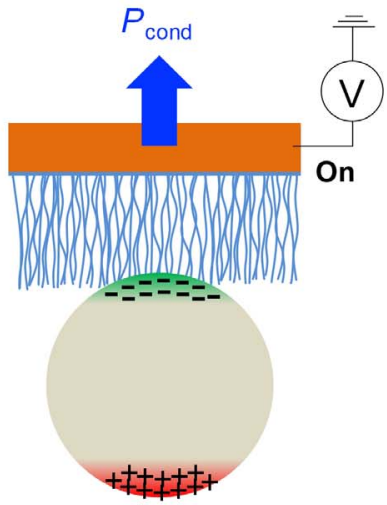

Conductive polarization

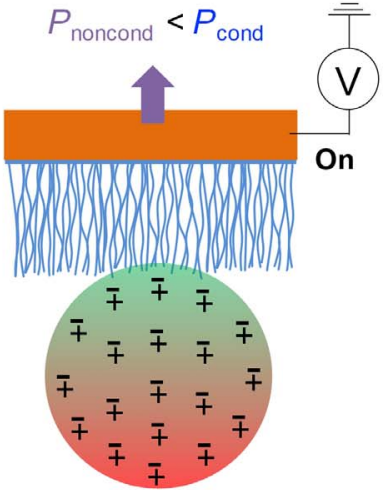

Dielectric polarization

Fig. 3. Switchable adhesion influenced by structural design and object conductivity. (A) Adhesion on/off ratio (ratio of pull-off force with an external voltage to that without voltage) of $\mathrm{Al}_{2} \mathrm{O}_{3}$-CNT SNE with different $\mathrm{Al}_{2} \mathrm{O}_{3}$ coating thicknesses (approximately $0.2,1$, and $10 \mathrm{~nm}$ ) and different fiber densities (sparse and dense). (B) AFM images (top) images of CNTs coated with $\mathrm{Al}_{2} \mathrm{O}_{3}$ via ALD with 10 (left) and 50 (right) deposition cycles, respectively, and SEM images (bottom) of SNE top surfaces with different densities. (C) Pull-off forces against a conductive (Pt-coated; $\sim 4 \mu \mathrm{m}$ diameter) and a nonconductive (uncoated $\mathrm{SiO}_{2} ; \sim 10 \mu \mathrm{m}$ diameter) spherical tip. (D) Schematics of polarization of conductive and dielectric objects when the SNE is in contact with the external voltage on.

Another important factor is the conductivity of the object being manipulated; in the above experiments, both the SNE and the object or probe tip were conductive, and one was grounded. As a charged $\mathrm{Al}_{2} \mathrm{O}_{3}$-CNT SNE approaches an object, the local electric field induces charge on the surface of the object. In conductive objects, mobile charges move to the surface to nullify the internal electric field. As a result, the electric field between the CNTs and the object is strong, as is the electrostatic force. In contrast, for nonconductive objects, charge is induced through polarization, i.e., by the electric displacement of bound electron clouds, stretching of polar bonds, and permanent dipole alignment (30). Compared to a conductive object, this results in a lower charge density at a given voltage and a weaker electrostatic force between the CNTs and the nonconductive object (Fig. 3B). However, the stronger electric field created between the SNE and a conductive object will mean that the dielectric coating on the conductive nanofibers will undergo dielectric breakdown at a lower voltage than with a nonconductive object. Thus, while, at a given voltage, the force on a nonconductive object is significantly lower than on a conductive object, significantly higher voltages can be applied to pick up nonconductive objects. This brings the achievable adhesion force for nonconductive objects to within the same range as conductive objects, at the expense of requiring higher voltages.
The scaling of electrostatic attraction compared to van der Waals forces on the SNE can be further understood from a contact mechanics model modified from classical theories (31). We derive the pull-off force $P_{\mathrm{SNE}}$ against a sphere having radius $R$ as (details in the Supplementary Materials)

$$
P_{\mathrm{SNE}}=F_{\mathrm{vdW}}+F_{\mathrm{es}}=\frac{3 \pi}{2} \eta \varphi \Delta \gamma R+\frac{\pi \varepsilon^{*}}{4 t^{* 2}}\left(\frac{9 \pi R^{2} \eta \varphi \Delta \gamma}{E^{*}}\right)^{2 / 3} V^{2}
$$

where $\eta$ is the fiber contact ratio; $\varphi$ is the fiber density; $\Delta \gamma$ is the work per unit area required to separate two solid smooth surfaces without externally induced electrical charges; $\varepsilon^{*}$ and $t^{*}$ are the effective permittivity and effective thickness of the dielectric coating and the air gap, respectively; and $E^{*}$ is the reduced modulus of the two contacting surfaces. As given in the first term in Eq. 1, the low fiber contact ratio $(0<$ $\eta<1)$ and the low fiber density ( $\sim 0.1$ or less) are the two major factors that enable the low intrinsic adhesion of the SNE (the first term of the equation). The fiber contact ratio within the nominal contact area depends on the applied load, fiber height variation, and the target object roughness, as owing to height variation, only the most distal nanofiber tips make initial contact (32). Our experiments show that SNE adhesion 

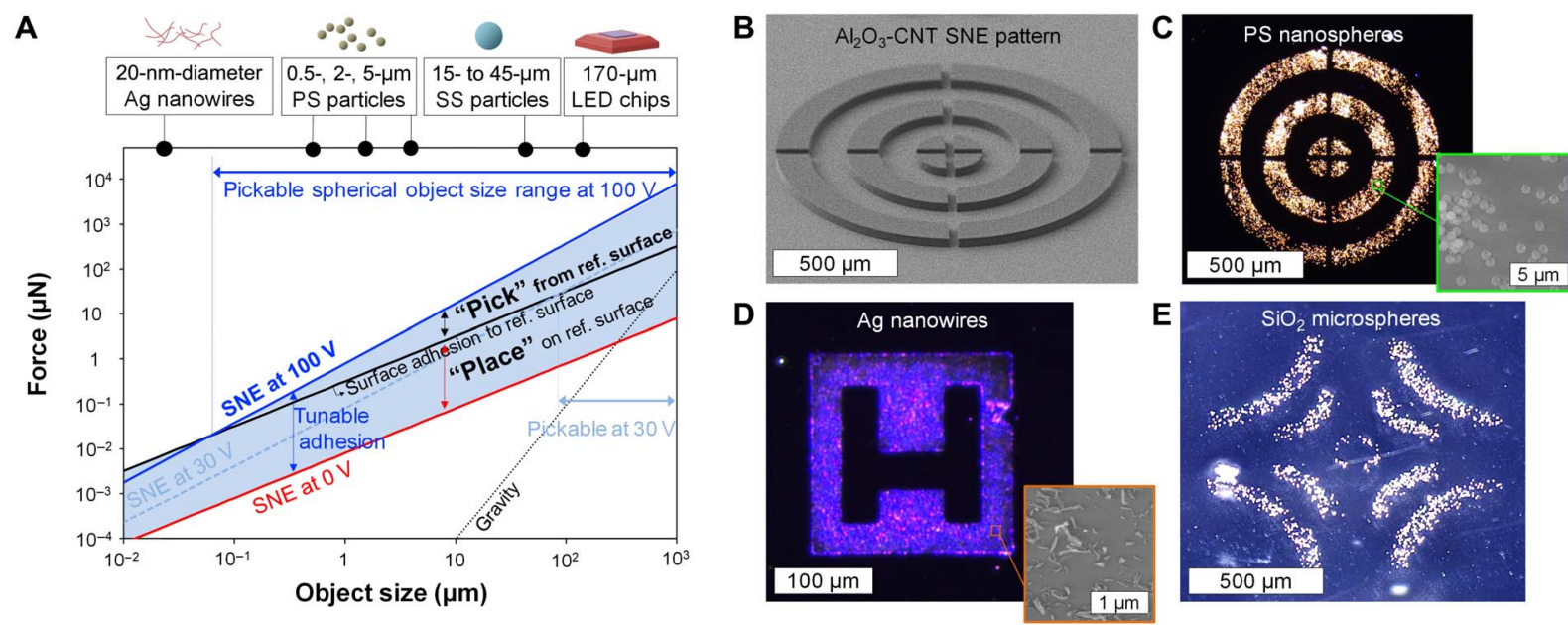

Fig. 4. Manipulation of multiple micro/nano objects using patterned SNE. (A) A micro-pick-and-place map showing the capable object size range that can be picked (by the adhesion force estimated from Eq. 1; blue solid and dashed lines) and placed (estimated from the first term of Eq. 1; red solid line) against a general flat surface with the same coating material (estimated from eq. S2; black solid line) at different operating voltages (30 and $100 \mathrm{~V}$ ). The dots on the top $x$ axis are the demonstrated materials in this study. (B) An SEM image $\left(60^{\circ}\right.$ tilted) of a micropatterned $\mathrm{Al}_{2} \mathrm{O}_{3}-\mathrm{CNT}$ SNE. (C) Optical microscope images of transfer printed polystyrene (PS) microspheres (500 nm in diameter) and (D) Ag nanowires ( $20 \mathrm{~nm}$ in diameter and $<12 \mu \mathrm{m}$ in length) on a $\mathrm{SiO}_{2}$ (thermally oxidized, $300 \mathrm{~nm}$ )/Si wafer. (E) An optical microscope image $\left(30^{\circ}\right.$ tilted) of $5-\mu \mathrm{m} \mathrm{SiO}_{2}$ microspheres transfer printed on a polyethylene terephthalate (PET) film.

increases with preload (fig. S9), whereas the adhesion of solid surfaces is generally independent of preload (fig. S10). The model here only considered normal contact without any shear motion; we experimentally find a decrease in the pull-off force after a shear motion with applied voltages for a spherical object (fig. S11). Therefore, to achieve high maximum SNE adhesion, one should apply normal preload but prevent lateral slip at the interface.

Therefore, proper design of the SNE and understanding of its mechanism of operation enables its use for ambient, electrically switchable manipulation of ultrasmall spherical objects including electronic chiplets, microparticles, and engineered nanostructures. Using Eq. 1, we construct a map (Fig. 4A) that shows the size range of objects that can be manipulated using the demonstrated SNE. For an object of given size, the on-state adhesion of the SNE should exceed the intrinsic adhesion between the object and the donor surface; thus, its size should fall in the "pick" regime. The off-state adhesion should be less than the intrinsic adhesion between the object and the target surface; thus, its size should fall in the "place" regime. For example, we predict that $30 \mathrm{~V}$ applied to the $\mathrm{Al}_{2} \mathrm{O}_{3}$-CNT SNE can pick an object with a size of $\sim 10$ to $100 \mu \mathrm{m}$; this is verified by the experiment with a $170 \mu \mathrm{m}$ by $170 \mu \mathrm{m}$ by $50 \mu \mathrm{m}$ micro-LED chiplet (Fig. 1C).

The adhesion measurements and scaling suggest that higher applied voltages $(100 \mathrm{~V})$ allow the SNE to pick objects in the far submicrometer size regime. Hence, we demonstrate the use of lithographically patterned SNEs to print complex patterns of micro- and nanomaterials, shown in Fig. 4B, including polymer beads (Fig. 4C) and films of silver nanowires (Fig. 4D). Here, both the donor and target substrates were rigid $\mathrm{SiO}_{2} / \mathrm{Si}$ wafers, therefore requiring the $\mathrm{SNE}$ object adhesion to be switchable to above and below this starting and ending condition. Uniform printing matching the complex shape of the SNE is obtained by simply turning on the DC voltage for pickup and turning off the voltage after the objects are placed in the desired transfer location. However, transfer is not deterministic as some objects transfer without voltage applied to the SNE during pickup (fig. S15). This is attributed to the nonuniformity of the donor layer of objects, which is created by spin coating. Printing is also demonstrated onto flexible polymer films, as in Fig. 4E, which is easier because polymers have stronger surface adhesion than rigid ceramics or metals. The silver nanowires, particularly having a diameter of $\sim 20 \mathrm{~nm}$, could successfully be picked although they are not in the pickable spherical object range. This is due to their wavy structure that induces point contacts (nanometer-scale spherical contacts), while the long-range electrostatic attraction is effective on the whole length of the wire.

In conclusion, the capability of soft, nanostructured surfaces to electrically switch their dry adhesion against both metallic and dielectric objects may provide a deft alternative to emerging transfer printing technologies that require thermally or chemically switchable adhesion. Moreover, SNEs may also advance biomimicry of natural surfaces that use polymer-like materials and complex mechanical motions to establish strong adhesion for locomotion. Lithographic patterning of SNE "pixels" in arbitrary patterns, along with their electrical addressability, may enable digital stamps for massive-scale micro- and nanoscale nextgeneration optical and electronic devices. In addition, SNEs based on 3D CNT microarchitectures $(28,29)$ could be used to build active legs and pads for miniature climbing robots (33) and microgripper arrays. Applications of SNEs in macroscale manipulation may also be possible, as the measured maximum adhesive strength (force per area) of the SNE (up to $\sim 20 \mathrm{kPa}$ at $225 \mathrm{~V}$ ) is comparable to planar electroadhesives (at $>5 \mathrm{kV})(20)$. However, robustness over many cyclic contacts and the influence of charge accumulation and dissipation on the maximum speed of operation require further verification for industrialization.

\section{MATERIALS AND METHODS \\ Fabrication of SNE}

A 50-nm TiN layer was first deposited by sputtering physical vapor deposition (PVD; Endura 5500, Applied Materials) on 4" (100) silicon wafers with $300 \mathrm{~nm}$ of thermally grown $\mathrm{SiO}_{2}$. Then, an $\mathrm{Al}_{2} \mathrm{O}_{3} / \mathrm{Fe}$ catalyst layer was patterned on top of the TiN layer, by lift-off processing using photolithography, followed by ultrasonic agitation in acetone. 
The supported catalyst layer, $10 \mathrm{~nm}$ of $\mathrm{Al}_{2} \mathrm{O}_{3}$ and $1 \mathrm{~nm}$ of $\mathrm{Fe}$, was sequentially deposited by electron beam PVD (VES-2550, Temescal). The wafer with the deposited catalyst was diced into $\sim 2 \mathrm{~cm}$ by $2 \mathrm{~cm}$ pieces and placed in a quartz tube furnace for CNT growth. The growth recipe starts with flowing 100/400 SCCM (standard cubic centimeters per minute) of $\mathrm{He} / \mathrm{H}_{2}$ while heating the furnace up to $775^{\circ} \mathrm{C}$ over $10 \mathrm{~min}$ (ramping step) and then holds at $775^{\circ} \mathrm{C}$ for $10 \mathrm{~min}$ with the same gas flow rates (annealing step). Then, the gas flow is changed to 100/400/ $100 \mathrm{SCCM}$ of $\mathrm{C}_{2} \mathrm{H}_{4} / \mathrm{He} / \mathrm{H}_{2}$ at $775^{\circ} \mathrm{C}$ for $\mathrm{CNT}$ growth for the selected duration. The typical growth rate is $\sim 100 \mu \mathrm{m} / \mathrm{min}$, and the CNTs are multiwalled with $\sim 10$-nm mean diameter with 0.45 coefficient of variation at the top of the forest, as we have previously measured by $\mathrm{x}$-ray scattering (24). After the growth, the furnace is cooled down to $<100^{\circ} \mathrm{C}$ at the same gas flow and lastly purged with 1000 SCCM of He for $5 \mathrm{~min}$. Last, $\mathrm{Al}_{2} \mathrm{O}_{3}$ was deposited onto CNTs by ALD (GEMStar, Arradiance Corporation). Trimethylaluminum (TMA) and ozone $\left(\mathrm{O}_{3}\right)$ were used as the metalorganic and oxidizing precursors, respectively. Using nitrogen as the carrier gas at a flow rate of 40 SCCM, TMA and $\mathrm{O}_{3}$ were sequentially pulsed into the deposition chamber ( 2 to 3 torr at $175^{\circ} \mathrm{C}$ ) for 22 and $100 \mathrm{~ms}$, respectively. Following each precursor pulse, the chamber was purged with 90 SCCM of nitrogen for 38 s. Polyimide tapes were used to mask the electrical contact points.

\section{Adhesion tests (AFM)}

The adhesion tests for a conductive material were done using a standard AFM (Bruker ICON AFM) in conductive mode. The samples were mounted by a vacuum holder and electrically connected to the steel stage. A platinum-coated AFM probe was mounted to the tip holder and connected to an electric control module. The module controlled the voltage bias between the steel stage and the AFM probe. A schematic of the setup is shown in fig. S3. The AFM probes are specially developed products (SD-Sphere-CONT from Nanosensors) with a sphere $(\sim 4.5 \mu \mathrm{m}$ in diameter $)$ at the tip and coated with platinum $(\sim 140 \mathrm{~nm})$ by PVD. Before the experiments, the stiffness of the AFM cantilever was calibrated using a thermal tuning method. During each experiment, voltage bias was held constant. The adhesion measurements were performed by bringing the tip into contact with the nanocomposite sample until a preset normal load was reached and then retracting the tip. The maximum adhesive force observed in the force-displacement curve during retraction was recorded. The approach and retraction speeds were fixed at $500 \mathrm{~nm} / \mathrm{s}$. The adhesion tests were repeated in multiple locations on samples, and the preset voltage bias and preload were varied in a random sequence for different experiments. The adhesion tests for nonconductive material were done using a standard AFM (Park Systems XE7). The samples were mounted on a magnetic holder and electrically connected to the base. The AFM probe was a tipless cantilever (ACLA-TL) onto which a $\mathrm{SiO}_{2}(10$ to $15 \mu \mathrm{m})$ particle was attached. The probe was mounted to the tip holder and electrically connected to the AFM head. Before the experiments, the stiffness of the AFM cantilever was estimated using a thermal tuning method. During each experiment, voltage bias, approach and retraction speed, and the preload were held constant. The adhesion measurements were performed by bringing the tip into contact with the CNT sample until a preset normal load was reached and then the tip was retracted. The maximum adhesive force observed in the force-displacement curves during retraction was recorded. The approach and retraction speeds were fixed at $300 \mathrm{~nm} / \mathrm{s}$. The adhesion tests were repeated at multiple locations on samples, and the preset voltage bias and preload were varied for different experiments.

\section{Millimeter-scale force measurements}

Millimeter-scale force measurements on the nanocomposite electroadhesives were performed using custom-built equipment. This equipment used two single-component load cells (LRM200-FSH02899, Futek; \pm 100 g capacity) attached to a flexure mechanism that decoupled the horizontal and vertical components of force. Attached to the flexure was a flat-tipped probe consisting of a 1.2-mm square silicon wafer die (X040093000W, WaferPro; 300-nm thermal oxide layer, diced on DISCO DAD-3240 diesaw) mounted on a slender aluminum column. The entire probe was sputter-coated with gold (100 nm; MS Q150T ES coater, Quorum) for electrical continuity. Voltage was applied between the tip and CNT forest through copper tape connected to the base of the probe and the forest away from the area of force measurements. The CNT forest sample was leveled relative to the tip on a tip-tilt-rotation stage (TTR001, Thorlabs). The load cell, flexure, and probe assembly was mounted on a two-axis stepper motor-controlled traversing mechanism capable of $100-\mathrm{nm}$ vertical resolution and $1-\mu \mathrm{m}$ lateral resolution.

Attractive force measurements were performed by positioning the tip at least $30 \mu \mathrm{m}$ above the CNT forest, applying $100 \mathrm{~V}$ (PS375/ $+20 \mathrm{kV}$, Stanford Research Systems) between them, and then approaching the surface at a speed of $0.5 \mu \mathrm{m} / \mathrm{s}$. Force measurements were collected every $100 \mathrm{~nm}$ until contact was made with the CNTs. Adhesion measurements were performed by positioning the tip above the CNT forest and approaching the surface at $0.2 \mu \mathrm{m} / \mathrm{s}$ without voltage applied. Tip motion was stopped once the desired preload force was reached. Voltage was then applied, and the tip was retracted at a speed of $0.2 \mu \mathrm{m} / \mathrm{s}$. The maximum tensile force recorded during retraction was reported as the adhesion force.

\section{Pick-and-place demonstration}

Pick-and-place demonstration of micro-LEDs and stainless steel microparticles (fig. S16) was done on a custom micromechanical test system consisting of a stepper motor-driven stage for controlling motion in the $z$ direction and a two-axis manual translation stage for controlling motion in the $x$ and $y$ directions. The SNE was mounted on a force sensor with a capacity of $100 \mathrm{mN}$. Voltages up to $30 \mathrm{~V}$ were applied with a source meter (Keithley 2400). The experiment was viewed from the side with a long working distance microscope (Navitar) and a complementary metal-oxide semiconductor camera (D722MU, Pixelink). For LED pick and place, a $200 \mu \mathrm{m}$ by $200 \mu \mathrm{m}$ SNE was contacted to the LED chiplet that was resting on a Si substrate; the load cell was used to control the preload. After contact, a voltage of $30 \mathrm{~V}$ was applied to the bottom TiN layer of the SNE relative to a ground connected to the stage supporting the Si substrate. The SNE was retracted upward, retrieving the LED. The SNE with the LED was then brought back into contact with the wafer surface, and the voltage was turned off. Upon retraction of the SNE, the SNE separated from the LED with no measurable force. The scale bar is $200 \mu \mathrm{m}$. For multiple microparticles and nanowires, a custom-made manual stage was used. First, the microparticle or nanowire dispersion was spin-coated on an $\mathrm{Al}_{2} \mathrm{O}_{3}$-coated Si wafer at $>1500 \mathrm{rpm}$ for $>5 \mathrm{~min}$. The particle/wire layer was further dried in an atmospheric environment for $>1$ day. A patterned $\mathrm{Al}_{2} \mathrm{O}_{3^{-}}$ CNT SNE was brought into contact against the particle/wire layer. A customized manual stage was used to gently bring the nanocomposite onto the layer and be pressed by its own weight. As the spin-coated particle layer was not uniform, a slight lateral motion (few micrometers) was induced to allow most of the SNE surface to make intimate contact with the particles. Then, an external voltage of $>40 \mathrm{~V}$ was applied by a power supply into the bottom TiN layer attached to the SNE against a 
ground electrode. The SNE was lifted up, while the voltage is still on. The target substrate (e.g., Si wafer, glass plate, and polyethylene terephthalate substrate) was placed underneath, and then, the SNE was again brought down gently onto the target substrate. Last, we removed the SNE after turning off the voltage.

\section{SUPPLEMENTARY MATERIALS}

Supplementary material for this article is available at http://advances.sciencemag.org/cgi/ content/full/5/10/eaax4790/DC1

Supplementary Text

Fig. S1. Schematics of the $\mathrm{Al}_{2} \mathrm{O}_{3}$-CNT SNE fabrication procedure.

Fig. S2. Diagram and optical images of unpackaged LED chiplets (UT170-31, CREE) used in picking and placing demonstration.

Fig. S3. Atomic force microscopy (AFM) system used for indentation tests with external voltages.

Fig. S4. Adhesion on/off ratio (ratio of pull-off force with an external voltage to that without voltage) of $\mathrm{Al}_{2} \mathrm{O}_{3}$-coated surfaces with and without SNE on a TiN layer.

Fig. S5. Optical microscope images of a paper towel showing its microscopically porous and rough surface.

Fig. S6. Transmission electron microscopy images of $\mathrm{Al}_{2} \mathrm{O}_{3}$ coating on individual CNT fibers via ALD with different numbers of cycles.

Fig. S7. $\mathrm{Al}_{2} \mathrm{O}_{3}$-CNT SNEs grown with different CVD procedures, exhibiting different CNT densities.

Fig. S8. Pull-off forces of $\mathrm{Al}_{2} \mathrm{O}_{3}$-CNT SNEs with different heights according to applied voltages. Fig. S9. Pull-off forces of $\mathrm{Al}_{2} \mathrm{O}_{3}$-CNT SNEs measured by AFM using a $4 \mu \mathrm{m}$ Pt-coated spherical tip with different applied external voltages and preloads.

Fig. S10. Pull-off forces of $\mathrm{Al}_{2} \mathrm{O}_{3}$ coated TiN layer measured by AFM using a $4 \mu \mathrm{m}$ Pt-coated spherical tip with different applied external voltages and preloads.

Fig. S11. Pull-off forces of SNE measured by AFM indentation using a $10-\mu \mathrm{m} \mathrm{SiO}_{2}$ spherical tip with and without the shear motion.

Fig. S12. Schematics of adhesion tests on an $\mathrm{Al}_{2} \mathrm{O}_{3}-\mathrm{TiN}$ surface using a microsphere.

Fig. S13. Contact mechanics modeling of adhesion between an $\mathrm{Al}_{2} \mathrm{O}_{3}-\mathrm{CNT}$ nanocomposite surface and a microsphere.

Fig. S14. Adhesive and gravitational forces along the object size.

Fig. S15. Optical microscope images of PS microspheres picked from a spin-coated layer using a patterned SNE surface and placed on a silicon wafer surface, under on or off electrical voltage conditions.

Fig. S16. Picking-and-placing of stainless steel (SS) microparticles, sized 15-45 $\mu \mathrm{m}$, using a $200 \mu \mathrm{m}$ by $200 \mu \mathrm{m} \mathrm{Al} \mathrm{O}_{3}$-CNT SNE, from a silicon wafer to a transparent substrate. Movie S1. Picking and placing of an unpackaged micro-LED chiplet using an $\mathrm{Al}_{2} \mathrm{O}_{3}$-CNT nanocomposite electroadhesive (side view).

Movie S2. Picking and placing of a piece of paper towel using an $\mathrm{Al}_{2} \mathrm{O}_{3}-\mathrm{CNT}$ nanocomposite electroadhesive and polydimethylsiloxane block.

\section{REFERENCES AND NOTES}

1. D. V. Dao, K. Nakamura, T. T. Bui, S. Sugiyama, Micro/nano-mechanical sensors and actuators based on SOI-MEMS technology. Adv. Nat. Sci. Nanosci. Nanotechnol. 1, 013001 (2010).

2. F. Alimenti, M. Virili, G. Orecchini, P. Mezzanotte, V. Palazzari, M. M. Tentzeris, L. Roselli, A new contactless assembly method for paper substrate Antennas and UHF RFID chips. IEEE Trans. Microw. Theory Tech. 59, 627-637 (2011).

3. K. Chung, H. Yoo, J. K. Hyun, H. Oh, Y. Tchoe, K. Lee, H. Baek, M. Kim, G.-C. Yi, Flexible GaN light-emitting diodes using $\mathrm{GaN}$ microdisks epitaxial laterally overgrown on graphene dots. Adv. Mater. 28, 7688-7694 (2016).

4. A. Carlson, A. M. Bowen, Y. Huang, R. G. Nuzzo, J. A. Rogers, Transfer printing techniques for materials assembly and micro/nanodevice fabrication. Adv. Mater. 24, 5284-5318 (2012).

5. E. Avci, K. Ohara, C.-N. Nguyen, C. Theeravithayangkura, M. Kojima, T. Tanikawa, Y. Mae, T. Arai, High-speed automated manipulation of microobjects using a two-fingered microhand. IEEE Trans. Ind. Electron. 62, 1070-1079 (2015).

6. W. Rong, Z. Fan, L. Wang, H. Xie, L. Sun, A vacuum microgripping tool with integrated vibration releasing capability. Rev. Sci. Instrum. 85, 085002 (2014).

7. A. N. Das, R. Murthy, D. O. Popa, H. E. Stephanou, A multiscale assembly and packaging system for manufacturing of complex micro-nano devices. IEEE Trans. Autom. Sci. Eng. 9, 160-170 (2012).

8. J. P. K. Seville, C. D. Willett, P. C. Knight, Interparticle forces in fluidisation: A review. Powder Technol. 113, 261-268 (2000).
9. R. S. Fearing, Survey Of Sticking Effects For Micro Parts Handling in Proceedings IEEE/RSJ International Conference on Intelligent Robots and Systems, 5 to 9 August 1995, pp. 212-217.

10. Y. Zhang, B. K. Chen, X. Liu, Y. Sun, Autonomous robotic pick-and-place of microobjects. IEEE Trans. Robot. 26, 200-207 (2010).

11. J. Chung, W. Zheng, T. J. Hatch, H. O. Jacobs, Programmable reconfigurable self-assembly: Parallel heterogeneous integration of chip-scale components on planar and nonplanar surfaces. J. Microelectromech. Syst. 15, 457-464 (2006).

12. W. Lu, A. M. Sastry, Self-assembly for semiconductor industry. IEEE Trans. Semiconductor Manufac. 20, 421-431 (2007).

13. A. Carlson, H.-J. Kim-Lee, J. Wu, P. Elvikis, H. Cheng, A. Kovalsky, S. Elgan, Q. Yu, P. M. Ferreira, Y. Huang, K. T. Turner, J. A. Rogers, Shear-enhanced adhesiveless transfer printing for use in deterministic materials assembly. Appl. Phys. Lett. 98, 264104 (2011).

14. S. Song, M. Sitti, Soft grippers using micro-fibrillar adhesives for transfer printing. Adv.Mater. 26, 4901-4906 (2014).

15. H. E. Jeong, J.-K. Lee, H. N. Kim, S. H. Moon, K. Y. Suh, A nontransferring dry adhesive with hierarchical polymer nanohairs. Proc. Natl. Acad. Sci. U.S.A. 106, 5639-5644 (2009).

16. J. D. Eisenhaure, T. Xie, S. Varghese, S. Kim, Microstructured shape memory polymer surfaces with reversible dry adhesion. ACS Appl. Mater. Interfaces 5, 7714-7717 (2013).

17. J. L. Wilbur, A. Kumar, E. Kim, G. M. Whitesides, Microfabrication by microcontact printing of self-assembled monolayers. Adv. Mater. 6, 600-604 (1994).

18. A. Bernard, J. P. Reneault, B. Michael, H. R. Bosshard, E. Delamarche, Microcontact printing of proteins. Adv. Mater. 12, 1067-1070 (2000).

19. J. Hesselbach, S. Buettgenbach, J. Wrege, S. Buetefisch, C. Graf, Centering electrostatic microgripper and magazines for microassembly. Proc. SPIE 4568, 270-277 (2001).

20. J. Shintake, S. Rosset, B. Schubert, Versatile soft grippers with intrinsic electroadhesion based on multifunctional polymer actuators. Adv. Mater. 28, 231-238 (2016).

21. S. Saito, F. Soda, R. Dhelika, K. Takahashi, W. Takarada, T. Kikutani, Compliant electrostatic chuck based on hairy microstructure. Smart Mater. Struc. 22, 015019 (2013).

22. K. Hata, D. N. Futaba, K. Mizuno, T. Namai, M. Yumura, S. lijima, Water-assisted highly efficient synthesis of impurity-free single-walled carbon nanotubes. Science 306, 1362-1364 (2004).

23. A. J. Hart, A. H. Slocum, Rapid growth and flow-mediated nucleation of millimeter-scale aligned carbon nanotube structures from a thin-film catalyst. J. Phys. Chem. B 110, 8250-8257 (2006).

24. A. M. Rao, D. Jacques, R. C. Haddon, In situ-grown carbon nanotube array with excellent field emission characteristics. Appl. Phys. Lett. 76, 3813-3815 (2000).

25. I. Lahiri, S.-M. Oh, J. Y. Hwang, C. Kang, M. Choi, H. Jeon, R. Banerjee, Y.-K. Sun, W. Choi, Ultrathin alumina-coated carbon nanotubes as an anode for high capacity Li-ion batteries. J. Mater. Chem. 12, 13621-13626 (2011).

26. M. Bedewy, E. R. Meshot, M. J. Reinker, A. J. Hart, Population growth dynamics of carbon nanotubes. ACS Nano 5, 8974-8989 (2011).

27. B. Lee, S.-Y. Park, H.-C. Kim, K. Cho, E. M. Vogel, M. J. Kim, R. M. Wallace, J. Kim, Conformal $\mathrm{Al}_{2} \mathrm{O}_{3}$ dielectric layer deposited by atomic layer deposition for graphene-based nanoelectronics. Appl. Phys. Lett. 92, 203102 (2008).

28. M. De Volder, S. Part, S. Tawfick, A. J. Hart, Strain-engineered manufacturing of freeform carbon nanotube microstructures. Nat. Commun. 5, 4512 (2014).

29. S. J. Park, H. Zhao, S. Kim, M. De Volder, A. J. Hart, Predictive synthesis of freeform carbon nanotube microarchitectures by strain-engineered chemical vapor deposition. Small 12, 4393-4403 (2016).

30. J. Hesselbach, S. Büettgenbach, J. Wrege, S. Büetefisch, C. Graf, Centering electrostatic microgripper and magazines for microassembly tasks. in Proc. of SPIE Microrobotics and Micromanipulation, vol. 4568, pp. 270-277, Newton, October 2001.

31. K. L. Johnson, Contact Mechanics (Cambridge Univ. Press, 1985).

32. S. Kim, H. Sojoudi, H. Zhao, D. Mariappan, G. H. McKinley, K. K. Gleason, A. J. Hart, Ultrathin high-resolution flexographic printing using nanoporous stamps. Sci. Adv. 2, e1601660 (2016).

33. S. D. de Rivaz, B. Goldberg, N. Doshi, K. Jayaram, J. Zhou, R. J. Wood, Inverted and vertical climbing of a quadrupedal microrobot using electroadhesion. Sci. Robot. 19, eaau3038 (2018).

Acknowledgments: We thank P. Kumar and V. Balakrishnan at the Indian Institute of Technology Mandi for transmission electron microscopy imaging and C. Owens for discussions and review of the manuscript. CNT catalyst deposition and patterning were performed at the MIT Microsystems Technology Laboratory (MTL). Nanoindentation was performed at the MIT Nano Mechanical Technology Laboratory (NanoLab). Electron microscopy was performed at the MIT Center for Materials Science and Engineering (CMSE). Funding: Financial support to S.K., N.N., C.Ca. and A.J.H. at MIT was provided by the NSF (CMMI-1463181), the Toyota Research Institute, and the MIT Institute for Soldier 
Nanotechnologies under contract W911NF-13-D-0001. Financial support for Y.J., K.L.T.T., K.T.T., and C.Ch. at U. Pennsylvania was provided by the NSF (CMMI-1463344 and CMMI-1663037). Financial support to H.Z. at MIT was provided by the MIT-Skoltech Next Generation Program. Financial support to C.J. at MIT was provided by Lockheed Martin Corporation. Author contributions: A.J.H. and S.K. conceived the idea. S.K., A.J.H., and K.T.T. coordinated the project. S.K., H.Z., and C.J. designed the SNE fabrication process. S.K. fabricated samples. Y.J., S.K., M.S.H.B., N.N., and C.Ca. characterized the SNEs. S.K., K.L.T.T., C.Ca., and C.Ch. performed pick-and-place demonstrations. S.K. developed the model and analyzed the data. S.K. and A.J.H. wrote the manuscript. All authors discussed the results and reviewed the manuscript. Competing interests: The authors declare that they have no competing interests. Data and materials availability: All data needed to evaluate the conclusions in the paper are present in the paper and/or the Supplementary Materials. Additional data related to this paper may be requested from the authors.

Submitted 26 March 2019

Accepted 14 September 2019

Published 11 October 2019

10.1126/sciadv.aax4790

Citation: S. Kim, Y. Jiang, K. L. Thompson Towell, M. S. H. Boutilier, N. Nayakanti, C. Cao, C. Chen, C. Jacob, H. Zhao, K. T. Turner, A. J. Hart, Soft nanocomposite electroadhesives for digital micro- and nanotransfer printing. Sci. Adv. 5, eaax4790 (2019). 


\section{ScienceAdvances}

\section{Soft nanocomposite electroadhesives for digital micro- and nanotransfer printing}

Sanha Kim, Yijie Jiang, Kiera L. Thompson Towell, Michael S. H. Boutilier, Nigamaa Nayakanti, Changhong Cao, Chunxu Chen, Christine Jacob, Hangbo Zhao, Kevin T. Turner and A. John Hart

Sci Adv 5 (10), eaax4790.

DOI: $10.1126 /$ sciadv.aax4790

ARTICLE TOOLS

SUPPLEMENTARY MATERIALS

REFERENCES

PERMISSIONS http://advances.sciencemag.org/content/5/10/eaax4790

http://advances.sciencemag.org/content/suppl/2019/10/07/5.10.eaax4790.DC1

This article cites 30 articles, 3 of which you can access for free http://advances.sciencemag.org/content/5/10/eaax4790\#BIBL

http://www.sciencemag.org/help/reprints-and-permissions

Copyright (C) 2019 The Authors, some rights reserved; exclusive licensee American Association for the Advancement of Science. No claim to original U.S. Government Works. Distributed under a Creative Commons Attribution NonCommercial License 4.0 (CC BY-NC). 